\title{
ON MONOTONE SOLUTIONS OF SOME CLASSES OF DIFFERENCE EQUATIONS
}

STEVO STEVIĆ

Received 17 December 2005; Accepted 27 February 2006

We describe a method for finding monotone solutions of some classes of difference equations converging to the corresponding equilibria. The method enables us to confirm three conjectures posed by the present author in a talk, which are extensions of three conjectures by M. R. S. Kulenović and G. Ladas, Dynamics of Second Order Rational Difference Equations. With Open Problems and Conjectures. Chapman and Hall/CRC, 2002. It is interesting that the method, in some cases, can be applied also when the parameters are variable.

Copyright (C) 2006 Stevo Stević. This is an open access article distributed under the Creative Commons Attribution License, which permits unrestricted use, distribution, and reproduction in any medium, provided the original work is properly cited.

\section{Introduction}

Recently there has been a great interest in studying nonlinear difference equations of order greater than one. Many of these equations stem from mathematical biology, economy, population dynamics, and so forth (see, e.g., $[5,7-9,11,14]$ and the references therein). An interesting problem in the theory of difference equations is finding monotone solutions. This paper is devoted to this problem.

Motivated by [8, Conjectures 5.4.6 and 6.10.3] in a talk (see, [16]) we posed the following three conjectures. The first one concerns a generalization of (1.2).

Conjecture 1.1. Show that for every $p>-1$, the following equation:

$$
x_{n+1}=p+\frac{x_{n-k}}{\sum_{i=0}^{k-1} \alpha_{i} x_{n-i}}, \quad n=0,1, \ldots
$$

where $k \in \mathbb{N}, \alpha_{i} \geq 0, i=0, \ldots, k-1$, and $\sum_{i=0}^{k-1} \alpha_{i}=1$, has a positive solution which remains above the equilibrium $\bar{x}_{1}=p+1$ for all $n \geq-k$. 
2 Monotone solutions of difference equations

In [6] DeVault et al. investigate the behavior of the positive solutions of the difference equation

$$
x_{n+1}=p+\frac{x_{n-k}}{x_{n}}, \quad n=0,1, \ldots,
$$

where $p>0$ and $k \in \mathbb{N}$ is fixed. Among other things they have proved that all nonoscillatory solutions of (1.2) converge to the positive equilibrium $\bar{x}=p+1$.

Based on this observation they have posed the following open problem.

Open problem 1.2. Do there exist nonoscillatory solutions of (1.2)?

The following conjectures are generalizations of [8, Conjectures 5.4.6 and 6.10.3].

Conjecture 1.3. Show that the following equation:

$$
x_{n+1}=\frac{1+x_{n-k}}{\sum_{i=0}^{k-1} \alpha_{i} x_{n-i}}, \quad n=0,1, \ldots,
$$

where $k \in \mathbb{N}, \alpha_{i} \geq 0, i=0, \ldots, k-1$, and $\sum_{i=0}^{k-1} \alpha_{i}=1$, has a nontrivial positive solution which decreases to the equilibrium $x_{2}=(1+\sqrt{5}) / 2$.

Conjecture 1.4. Show that the following equation:

$$
x_{n+1}=\frac{\alpha+x_{n-k}}{1+\sum_{i=0}^{k-1} \alpha_{i} x_{n-i}}, \quad n=0,1, \ldots
$$

where $k \in \mathbb{N}, \alpha>0, \alpha_{i} \geq 0, i=0, \ldots, k-1$, and $\sum_{i=0}^{k-1} \alpha_{i}=1$, has a positive solution which decreases to the equilibrium $x_{3}=\sqrt{\alpha}$.

Our aim in this paper is to confirm the above mentioned conjectures.

The linearized equation for (1.1), respectively, (1.3) and (1.4), about the corresponding positive equilibrium $\bar{x}_{i}, i \in\{1,2,3\}$, is

$$
\begin{gathered}
(p+1) y_{n+1}+\alpha_{0} y_{n}+\cdots+\alpha_{k-1} y_{n-k+1}-y_{n-k}=0, \\
\bar{x}_{2}\left(y_{n+1}+\alpha_{0} y_{n}+\cdots+\alpha_{k-1} y_{n-k+1}\right)-y_{n-k}=0, \\
(1+\sqrt{\alpha}) y_{n+1}+\sqrt{\alpha}\left(\alpha_{0} y_{n}+\cdots+\alpha_{k-1} y_{n-k+1}\right)-y_{n-k}=0 .
\end{gathered}
$$

The characteristic polynomial associated with (1.5), respectively, (1.6) and (1.7), is

$$
\begin{gathered}
p_{1}(t)=(p+1) t^{k+1}+\alpha_{0} t^{k}+\cdots+\alpha_{k-1} t-1=0, \\
p_{2}(t)=\bar{x}_{2}\left(t^{k+1}+\alpha_{0} t^{k}+\cdots+\alpha_{k-1} t\right)-1=0, \\
p_{3}(t)=(1+\sqrt{\alpha}) t^{k+1}+\sqrt{\alpha}\left(\alpha_{0} t^{k}+\cdots+\alpha_{k-1} t\right)-1=0 .
\end{gathered}
$$

Since $p_{1}(0)=-1<0, p_{1}(1)=p+1$, and $p_{1}^{\prime}(t)=(p+1)(k+1) t^{k}+\alpha_{0} k t^{k-1}+\cdots+$ $\alpha_{k-1}>0$ for $t \in(0,1]$, it follows that for each $p>-1$, there is a unique positive root $t_{1}$ of the polynomial (1.8) belonging to the interval $(0,1)$. 
Similarly, it can be shown that (1.9) and (1.10) have also a unique positive roots $t_{2}$ and $t_{3}$ in the interval $(0,1)$.

This fact motivated us to believe that there are solutions of (1.1), (1.3), and (1.4) which have the following asymptotics:

$$
x_{n}=\bar{x}+a t_{i}^{n}+o\left(t_{i}^{n}\right)
$$

where $a \in \mathbb{R}$ and $t_{i}, i \in\{1,2,3\}$, are the above mentioned roots of polynomials (1.5), (1.6), and (1.7), respectively .

We solve the open problem, showing that such solutions exist, developing Berg's idea in [2] which are based on asymptotics. Asymptotics for solutions of difference equations has been investigated for a long time by L. Berg and S. Stević, see, for example, $[1-4,10-$ $15]$ and the reference therein. We solve it by constructing two appropriate sequences $y_{n}$ and $z_{n}$ with

$$
y_{n} \leq x_{n} \leq z_{n}
$$

for sufficiently large $n$. In $[1,2]$, some methods can be found for the construction of these bounds, see, also [3,4].

From (1.11) and results in Berg's paper [2], we expect that for $k \geq 2$ such solutions have the first four members in their asymptotics in the following form:

$$
\varphi_{n}=\bar{x}+a t^{n}+b t^{2 n}+c t^{3 n} .
$$

\section{The inclusion theorem}

We need the following result in the proof of the main theorem. The proof of the result is similar to that of [2, Theorem 1].

THeOREM 2.1. Let $f: I^{k+2} \rightarrow I$ be a continuous and nondecreasing function in each argument on the interval $I \subset \mathbb{R}$, and let $\left(y_{n}\right)$ and $\left(z_{n}\right)$ be sequences with $y_{n}<z_{n}$ for $n \geq n_{0}$ and such that

$$
y_{n-k} \leq f\left(n, y_{n-k+1}, \ldots, y_{n+1}\right), \quad f\left(n, z_{n-k+1}, \ldots, z_{n+1}\right) \leq z_{n-k},
$$

for $n>n_{0}+k-1$.

Then there is a solution of the following difference equation:

$$
x_{n-k}=f\left(n, x_{n-k+1}, \ldots, x_{n+1}\right) \text {, }
$$

with property (1.12) for $n \geq n_{0}$.

Proof. Let $N$ be an arbitrary integer such that $N>n_{0}+k-1$. The solution $\left(x_{n}\right)$ of (2.2) with given initial values $x_{N}, x_{N+1}, \ldots, x_{N+k}$ satisfying (1.12) for $n \in\{N, N+1, \ldots, N+k\}$ can be continued by (2.2) to all $n<N$. Inequalities (2.1) and the monotonic character of $f$ imply that (1.12) holds for all $n \in\left\{n_{0}, \ldots, N+k\right\}$. Let $A_{N}$ be the set of all $(k+1)$ tuples $\left(x_{n_{0}}, \ldots, x_{n_{0}+k}\right)$ such that there exist solutions $\left(x_{n}\right)$ of $(2.2)$ with these initial values satisfying (1.12) for all $n \in\left\{n_{0}, \ldots, N+k\right\}$. It is clear that $A_{N}$ is a closed nonempty set 
for every $N>n_{0}+k-1$, and that $A_{N+1} \subset A_{N}$. It follows that the set $A=\cap_{n=n_{0}+k}^{\infty} A_{N}$ is a nonempty subset of $\mathbb{R}^{k+1}$ and that if $\left(x_{n_{0}}, \ldots, x_{n_{0}+k}\right) \in A$, then the corresponding solutions of (2.2) satisfy (1.12) for all $n \geq n_{0}$, as desired.

\section{The main result}

In this section we prove the main result of this paper, which confirms Conjectures 1.1, 1.3 , and 1.4 .

Theorem 3.1. The following statements are true:

(a) let $\alpha_{i} \geq 0, i=0, \ldots, k-1, \sum_{i=0}^{k-1} \alpha_{i}=1$, and $p>-1$. Then (1.1) has a positive solution which remains above the equilibrium $\bar{x}_{1}=p+1$;

(b) let $\alpha_{i} \geq 0, i=0, \ldots, k-1, \sum_{i=0}^{k-1} \alpha_{i}=1$. Then (1.3) has a nontrivial positive solution which decreases to the equilibrium $\bar{x}_{2}$;

(c) let $\alpha>0, \alpha_{i} \geq 0, i=0, \ldots, k-1, \sum_{i=0}^{k-1} \alpha_{i}=1$. Then (1.4) has a nontrivial positive solution which decreases to the equilibrium $\bar{x}_{3}=\sqrt{\alpha}$.

Proof. (a) Note that (1.2) can be written in the following equivalent form:

$$
F\left(x_{n-k}, \ldots, x_{n}, x_{n+1}\right)=\left(x_{n+1}-p\right)\left(\alpha_{0} x_{n}+\cdots+\alpha_{k-1} x_{n-k+1}\right)-x_{n-k}=0 .
$$

We expect that solutions of (1.2) have asymptotic approximation (1.13). Thus, we calculate $F\left(\varphi_{n-k}, \ldots, \varphi_{n}, \varphi_{n+1}\right)$. We have

$$
\begin{aligned}
F= & \left(1+a t^{n+1}+b t^{2(n+1)}+c t^{3(n+1)}\right) \\
& \times\left(p+1+a \alpha_{0} t^{n}+\cdots+a \alpha_{k-1} t^{n-k+1}+b \alpha_{0} t^{2 n}+\cdots+b \alpha_{k-1} t^{2(n-k+1)}+\mathcal{O}\left(t^{3 n}\right)\right) \\
& -\left(p+1+a t^{n-k}+b t^{2(n-k)}+c t^{3(n-k)}\right) \\
= & a t^{n}\left((p+1) t+\alpha_{0}+\cdots+\frac{\alpha_{k-1}}{t^{k-1}}-t^{-k}\right) \\
& +t^{2 n}\left(b\left(\alpha_{0}+\cdots+\frac{\alpha_{k-1}}{t^{2(k-1)}}\right)+a^{2} t\left(\alpha_{0}+\cdots+\frac{\alpha_{k-1}}{t^{k-1}}\right)+b(p+1) t^{2}-b t^{-2 k}\right)+O\left(t^{3 n}\right) .
\end{aligned}
$$

Let

$$
D_{1}(t)=(p+1) t+\alpha_{0}+\cdots+\frac{\alpha_{k-1}}{t^{k-1}}-\frac{1}{t^{k}}
$$

Choose $t \in(0,1)$ such that $D_{1}(t)=0$, and $a, b \in \mathbb{R}, a \neq 0$, such that the coefficients in (3.2) are equal to zero. $D_{1}(t)=0$ implies that $t=t_{1}$ (see, Section 1). Further we obtain

$$
b=-\frac{a^{2} t_{1}\left(\alpha_{0}+\cdots+\alpha_{k-1} t_{1}^{-k+1}\right)}{(p+1) t_{1}^{2}+\alpha_{0}+\cdots+\left(\alpha_{k-1}\right) / t_{1}^{2(k-1)}-t_{1}^{-2 k}}=-\frac{a^{2} t_{1}\left(\alpha_{0}+\cdots+\alpha_{k-1} t_{1}^{-k+1}\right)}{D_{1}\left(t_{1}^{2}\right)}
$$


If $\hat{\varphi}_{n}=p+1+a t_{1}^{n}+q t_{1}^{2 n}$, we obtain

$$
F\left(\hat{\varphi}_{n-k}, \ldots, \hat{\varphi}_{n}, \hat{\varphi}_{n+1}\right) \sim\left(q D_{1}\left(t_{1}^{2}\right)+a^{2} t_{1}\left(\alpha_{0}+\cdots+\alpha_{k-1} t_{1}^{-k+1}\right)\right) t_{1}^{2 n}
$$

Let

$$
H_{t_{1}}(q)=q D_{1}\left(t_{1}^{2}\right)+a^{2} t_{1}\left(\alpha_{0}+\cdots+\alpha_{k-1} t_{1}^{-k+1}\right) .
$$

We have

$$
D_{1}^{\prime}(t)=p+1+\frac{k}{t^{k+1}}-\frac{\alpha_{1}}{t^{2}}-\cdots-\frac{(k-1) \alpha_{k-1}}{t^{k}} .
$$

Hence, when $t \in(0,1)$, it follows that

$$
\begin{aligned}
D_{1}^{\prime}(t) & >p+1+\frac{k}{t^{k+1}}-\frac{\alpha_{1}+\cdots+(k-1) \alpha_{k-1}}{t^{k+1}} \\
& >p+1+\frac{k}{t^{k+1}}-\frac{(k-1) \sum_{i=1}^{k_{1}} \alpha_{i}}{t^{k+1}}>p+1+\frac{1}{t^{k+1}}>0
\end{aligned}
$$

From this, since $D_{1}\left(t_{1}\right)=0$, and $t_{1}^{2}<t_{1}$, we have that $D_{1}\left(t_{1}^{2}\right)<0$. Thus, we obtain that there are $q_{1}<b$ and $q_{2}>b$ such that $H_{t_{1}}\left(q_{1}\right)>0$ and $H_{t_{2}}\left(q_{2}\right)<0$.

With the notations

$$
y_{n}=p+1+a t_{1}^{n}+q_{1} t_{1}^{2 n}, \quad z_{n}=p+1+a t_{1}^{n}+q_{2} t_{1}^{2 n},
$$

we get

$$
\begin{aligned}
& F\left(y_{n-k}, \ldots, y_{n}, y_{n+1}\right) \sim\left(q_{1} D_{1}\left(t_{1}^{2}\right)+a^{2} t_{1}\left(\alpha_{0}+\cdots+\alpha_{k-1} t_{1}^{-k+1}\right)\right) t_{1}^{2 n}>0, \\
& F\left(z_{n-k}, \ldots, z_{n}, z_{n+1}\right) \sim\left(q_{2} D_{1}\left(t_{1}^{2}\right)+a^{2} t_{1}\left(\alpha_{0}+\cdots+\alpha_{k-1} t_{1}^{-k+1}\right)\right) t_{1}^{2 n}<0 .
\end{aligned}
$$

These relations show that the inequalities in (1.12) are satisfied for sufficiently large $n$, where $f=F+x_{n-k}$ and $F$ is given by (3.1). Applying Theorem 2.1 it follows that there is a solution of (1.1) with the asymptotics $x_{n}=\hat{\varphi}_{n}+o\left(t_{1}^{2 n}\right)$, in particular, the solution of (1.1) converges monotonically to the positive equilibrium $\bar{x}_{1}=p+1$, when $p>-1$ and $n \geq n_{0}$. Hence, the solution $x_{n+n_{0}+k}$ converges monotonically for $n \geq-k$.

(b) Equation (1.3) can be written in the following equivalent form:

$$
F\left(x_{n-k}, \ldots, x_{n}, x_{n+1}\right)=x_{n+1}\left(\alpha_{0} x_{n}+\cdots+\alpha_{k-1} x_{n-k+1}\right)-\left(1+x_{n-k}\right)=0 .
$$


Now we calculate $F\left(\varphi_{n-k}, \ldots, \varphi_{n}, \varphi_{n+1}\right)$. We have

$$
\begin{aligned}
& F=\left(\bar{x}_{2}+a t^{n+1}+b t^{2(n+1)}+c t^{3(n+1)}\right) \\
& \times\left(\bar{x}_{2}+a \alpha_{0} t^{n}+\cdots+a \alpha_{k-1} t^{n-k+1}+b \alpha_{0} t^{2 n}+\cdots+b \alpha_{k-1} t^{2(n-k+1)}+\mathcal{O}\left(t^{3 n}\right)\right) \\
& -\left(1+\bar{x}_{2}+a t^{n-k}+b t^{2(n-k)}+c t^{3(n-k)}\right) \\
& =a t^{n}\left(\bar{x}_{2} t+\bar{x}_{2}\left(\alpha_{0}+\cdots+\frac{\alpha_{k-1}}{t^{k-1}}\right)-\frac{1}{t^{k}}\right) \\
& +t^{2 n}\left(\bar{x}_{2} b\left(\alpha_{0}+\cdots+\frac{\alpha_{k-1}}{t^{2(k-1)}}\right)+a^{2} t\left(\alpha_{0}+\cdots+\frac{\alpha_{k-1}}{t^{k-1}}\right)+\bar{x}_{2} b t^{2}-b t^{-2 k}\right)+\mathcal{O}\left(t^{3 n}\right) .
\end{aligned}
$$

Let

$$
D_{2}(t)=\bar{x}_{2} t+\bar{x}_{2}\left(\alpha_{0}+\cdots+\frac{\alpha_{k-1}}{t^{k-1}}\right)-t^{-k}=\frac{p_{2}(t)}{t^{k}} .
$$

Choose $t \in(0,1)$ such that $D_{2}(t)=0$, and $a, b \in \mathbb{R}, a \neq 0$, such that the coefficients in (3.12) are equal to zero. Since $D_{2}(t)=0$ is equivalent to $p_{2}(t)=0$, we have that $t=t_{2}$, and consequently

$$
b=-\frac{a^{2} t_{2}\left(\alpha_{0}+\cdots+\alpha_{k-1} t_{2}^{-k+1}\right)}{\bar{x}_{2} t_{2}^{2}+\bar{x}_{2}\left(\alpha_{0}+\cdots+\left(\alpha_{k-1}\right) / t_{2}^{2(k-1)}\right)-t_{2}^{-2 k}}=-\frac{a^{2} t_{2}\left(\alpha_{0}+\cdots+\alpha_{k-1} t_{2}^{-k+1}\right)}{D_{2}\left(t_{2}^{2}\right)} .
$$

If $\hat{\varphi}_{n}=\bar{x}_{2}+a t_{2}^{n}+q t_{2}^{2 n}$, we obtain

$$
F\left(\hat{\varphi}_{n-k}, \ldots, \hat{\varphi}_{n}, \hat{\varphi}_{n+1}\right) \sim\left(q D_{2}\left(t_{2}^{2}\right)+a^{2} t_{2}\left(\alpha_{0}+\cdots+\alpha_{k-1} t_{2}^{-k+1}\right)\right) t_{2}^{2 n} .
$$

Let

$$
H_{t_{2}}(q)=q D_{2}\left(t_{2}^{2}\right)+a^{2} t_{2}\left(\alpha_{0}+\cdots+\alpha_{k-1} t_{2}^{-k+1}\right) .
$$

Since

$$
p_{2}^{\prime}(t)=\bar{x}_{2}\left((k+1) t^{k}+k \alpha_{0} t^{k-1}+\cdots+\alpha_{k-1}\right)>0,
$$

when $t \in(0,1)$, and since $p_{2}\left(t_{2}\right)=0$, and $t_{2}^{2}<t_{2}$, we have that $p_{2}\left(t_{2}^{2}\right)<0$, which implies $D_{2}\left(t_{2}^{2}\right)<0$. Thus, we obtain that there are $q_{3}<b$ and $q_{4}>b$ such that $H_{t_{2}}\left(q_{3}\right)>0$ and $H_{t_{2}}\left(q_{4}\right)<0$.

With the notations

$$
y_{n}=\bar{x}_{2}+a t_{2}^{n}+q_{3} t_{2}^{2 n}, \quad z_{n}=\bar{x}_{2}+a t_{2}^{n}+q_{4} t_{2}^{2 n},
$$

we get

$$
\begin{aligned}
& F\left(y_{n-k}, \ldots, y_{n}, y_{n+1}\right) \sim H_{t_{2}}\left(q_{3}\right) t_{2}^{2 n}>0, \\
& F\left(z_{n-k}, \ldots, z_{n}, z_{n+1}\right) \sim H_{t_{2}}\left(q_{4}\right) t_{2}^{2 n}<0 .
\end{aligned}
$$


These relations show that the inequalities in (1.12) are satisfied for sufficiently large $n$, where $f=F+x_{n-k}$ and $F$ is given by (3.11). Applying Theorem 2.1 it follows that there is a solution of (1.3) with the asymptotics $x_{n}=\hat{\varphi}_{n}+o\left(t_{2}^{2 n}\right)$. This solution obviously converges monotonically to the positive equilibrium $\bar{x}_{2}=(\sqrt{5}+1) / 2$, for $n \geq n_{1}$. A suitable shift of $x_{n}$ is decreasing for all $n \geq-k$.

(c) Equation (1.4) can be written in the following equivalent form:

$$
F\left(x_{n-k}, \ldots, x_{n}, x_{n+1}\right)=x_{n+1}\left(1+\alpha_{0} x_{n}+\cdots+\alpha_{k-1} x_{n-k+1}\right)-\left(\alpha+x_{n-k}\right)=0 .
$$

We have

$$
\begin{aligned}
F= & \left(\bar{x}_{3}+a t^{n+1}+b t^{2(n+1)}+c t^{3(n+1)}\right) \\
& \times\left(1+\bar{x}_{3}+a \alpha_{0} t^{n}+\cdots+a \alpha_{k-1} t^{n-k+1}+b \alpha_{0} t^{2 n}+\cdots+b \alpha_{k-1} t^{2(n-k+1)}+\mathcal{O}\left(t^{3 n}\right)\right) \\
& -\left(\alpha+\bar{x}_{3}+a t^{n-k}+b t^{2(n-k)}+c t^{3(n-k)}\right) \\
= & a t^{n}\left(\left(1+\bar{x}_{3}\right) t+\bar{x}_{3}\left(\alpha_{0}+\cdots+\frac{\alpha_{k-1}}{t^{k-1}}\right)-t^{-k}\right) \\
& +t^{2 n}\left(\bar{x}_{3} b\left(\alpha_{0}+\cdots+\frac{\alpha_{k-1}}{t^{2(k-1)}}\right)+a^{2} t\left(\alpha_{0}+\cdots+\frac{\alpha_{k-1}}{t^{k-1}}\right)+\left(1+\bar{x}_{3}\right) b t^{2}-b t^{-2 k}\right) \\
& +O\left(t^{3 n}\right) .
\end{aligned}
$$

Let

$$
D_{3}(t)=\left(1+\bar{x}_{3}\right) t+\bar{x}_{3}\left(\alpha_{0}+\cdots+\frac{\alpha_{k-1}}{t^{k-1}}\right)-t^{-k}=\frac{p_{3}(t)}{t^{k}} .
$$

Choose $t \in(0,1)$ such that $D_{3}(t)=0$, and $a, b \in \mathbb{R}, a \neq 0$, such that the coefficients in (3.21) are equal to zero.

Since

$$
p_{3}^{\prime}(t)=(1+\sqrt{\alpha})(k+1) t^{k}+\sqrt{\alpha}\left(k \alpha_{0} t^{k-1}+\cdots+\alpha_{k-1}\right)>0,
$$

when $t \in(0,1]$, and $D_{3}(t)=0$ is equivalent to $p_{3}(t)=0$, we have that $t=t_{3}$. From this and (3.21) it follows that

$$
b=-\frac{a^{2} t_{3}\left(\alpha_{0}+\cdots+\alpha_{k-1} t_{3}^{-k+1}\right)}{(1+\sqrt{\alpha}) t_{3}^{2}+\sqrt{\alpha}\left(\alpha_{0}+\cdots+\left(\alpha_{k-1}\right) / t_{3}^{2(k-1)}\right)-t_{3}^{-2 k}}=-\frac{a^{2} t_{3}\left(\alpha_{0}+\cdots+\alpha_{k-1} t_{3}^{-k+1}\right)}{D_{3}\left(t_{3}^{2}\right)} .
$$

If $\hat{\varphi}_{n}=\sqrt{\alpha}+a t_{3}^{n}+q t_{3}^{2 n}$, we obtain

$$
F\left(\hat{\varphi}_{n-k}, \ldots, \hat{\varphi}_{n}, \hat{\varphi}_{n+1}\right) \sim\left(q D_{3}\left(t_{3}^{2}\right)+a^{2} t_{3}\left(\alpha_{0}+\cdots+\alpha_{k-1} t_{3}^{-k+1}\right)\right) t_{3}^{2 n} .
$$

Let

$$
H_{t_{3}}(q)=q D_{3}\left(t_{3}^{2}\right)+a^{2} t_{3}\left(\alpha_{0}+\cdots+\alpha_{k-1} t_{3}^{-k+1}\right) .
$$


Since $D_{3}\left(t_{3}\right)=0$, and $t_{3}^{2}<t_{3}$, we have that $D_{3}\left(t_{3}^{2}\right)<0$. Thus, we obtain that there are $q_{5}<b$ and $q_{6}>b$ such that $H_{t_{3}}\left(q_{5}\right)>0$ and $H_{t_{3}}\left(q_{6}\right)<0$.

With the notations

$$
y_{n}=\sqrt{\alpha}+a t_{3}^{n}+q_{5} t_{3}^{2 n}, \quad z_{n}=\sqrt{\alpha}+a t_{3}^{n}+q_{6} t_{3}^{2 n},
$$

we get

$$
F\left(y_{n-k}, \ldots, y_{n}, y_{n+1}\right) \sim H_{t_{3}}\left(q_{5}\right) t_{3}^{2 n}>0, \quad F\left(z_{n-k}, \ldots, z_{n}, z_{n+1}\right) \sim H_{t_{3}}\left(q_{6}\right) t_{3}^{2 n}<0 .
$$

These relations show that the inequalities in (1.12) are satisfied for sufficiently large $n$, where $f=F+x_{n-k}$ and $F$ is given by (3.20). Hence, there is a solution of (1.4) with the asymptotics $x_{n}=\hat{\varphi}_{n}+o\left(t_{3}^{2 n}\right)$. The result follows similarly to the above mentioned cases.

From Theorem 3.1(a) with $\alpha_{0}=1$ and $\alpha_{i}=0, i \neq 0$, we get the following corollary.

COROLlary 3.2. There is a nonoscillatory solution of (1.2).

Remark 3.3. Since $a \in \mathbb{R} \backslash\{0\}$ is an arbitrary parameter, by Theorem 3.1 we find a set of nonoscillatory solutions of (1.1), (1.3), and (1.4) converging to the corresponding positive equilibria.

Remark 3.4. Note that using (1.13) better asymptotics for these solutions can be obtained, that is, $x_{n}=\varphi_{n}+o\left(t_{i}^{3 n}\right), i \in\{1,2,3\}$, where $b$ is given by (3.4), (3.14), or (3.24), and $c$ can be found equating to zero the coefficient nearby $t^{3 n}$.

Remark 3.5. From the proof of Theorem 3.1, we see that we can assume that the parameter $p$ in (1.1) can be replaced by a nondecreasing sequence with the following asymptotics: $p_{n}=p+o\left(t_{1}^{2 n}\right)$.

\section{Acknowledgment}

I would like to express my sincere thanks to Professor L. Berg for giving valuable suggestions during the preparation of this paper.

\section{References}

[1] L. Berg, Asymptotische Darstellungen und Entwicklungen, Hochschulbücher für Mathematik, vol. 66, VEB Deutscher Verlag der Wissenschaften, Berlin, 1968.

[2] _ On the asymptotics of nonlinear difference equations, Zeitschrift für Analysis und ihre Anwendungen 21 (2002), no. 4, 1061-1074.

[3] Inclusion theorems for non-linear difference equations with applications, Journal of Difference Equations and Applications 10 (2004), no. 4, 399-408.

[4] Corrections to: "Inclusion theorems for non-linear difference equations with applications" from [3], Journal of Difference Equations and Applications 11 (2005), no. 2, 181-182.

[5] J. M. Cushing and Y. Kuang, Global stability in a nonlinear difference delay equation model of flour beetle population growth, Journal of Difference Equations and Applications 2 (1996), no. 1, 31-37.

[6] R. DeVault, C. Kent, and W. Kosmala, On the recursive sequence $x_{n+1}=p+\frac{x_{n-k}}{x_{n}}$, Journal of Difference Equations and Applications 9 (2003), no. 8, 721-730. 
[7] M. E. Fisher and B. S. Goh, Stability results for delayed-recruitment models in population dynamics, Journal of Mathematical Biology 19 (1984), no. 1, 147-156.

[8] M. R. S. Kulenović and G. Ladas, Dynamics of Second Order Rational Difference Equations. With Open Problems and Conjectures, Chapman and Hall/CRC, Florida, 2002.

[9] E. C. Pielou, Population and Community Ecology, Gordon and Breach, New York, 1974.

[10] S. Stević, Asymptotic behaviour of a sequence defined by iteration, Matematički Vesnik 48 (1996), no. 3-4, 99-105.

[11] Behavior of the positive solutions of the generalized Beddington-Holt equation, Panamerican Mathematical Journal 10 (2000), no. 4, 77-85.

[12] _ Asymptotic behavior of a sequence defined by iteration with applications, Colloquium Mathematicum 93 (2002), no. 2, 267-276.

[13] _ Asymptotic behaviour of a sequence defined by a recurrence formula. II, The Australian Mathematical Society Gazette 29 (2002), no. 4, 209-215.

[14] _ Asymptotic behavior of a nonlinear difference equation, Indian Journal of Pure and Applied Mathematics 34 (2003), no. 12, 1681-1687.

[15] On the recursive sequence $x_{n+1}=x_{n}+\frac{x_{n}^{\alpha}}{n^{\beta}}$, Bulletin of the Calcutta Mathematical Society 95 (2003), no. 1, 39-46.

[16] _ Some open problems and conjectures on difference equations, April 29, 2004, http:// www.mi.sanu.ac.yu.

Stevo Stević: Mathematical Institute of the Serbian Academy of Science Knez Mihailova 35/I, 11000 Beograd, Serbia

E-mail addresses: sstevic@ptt.yu; sstevo@matf.bg.ac.yu 


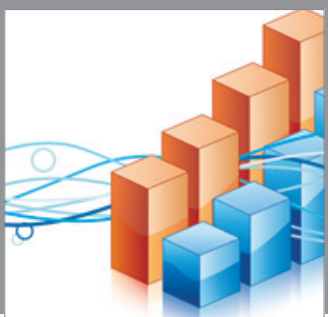

Advances in

Operations Research

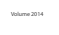

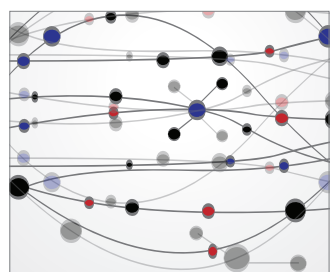

\section{The Scientific} World Journal
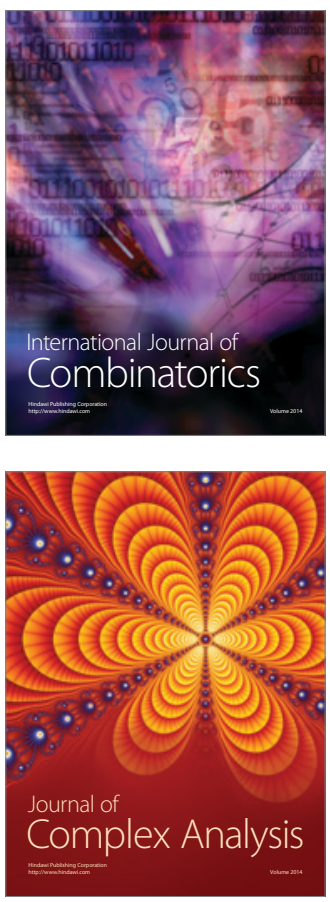

International Journal of

Mathematics and

Mathematical

Sciences
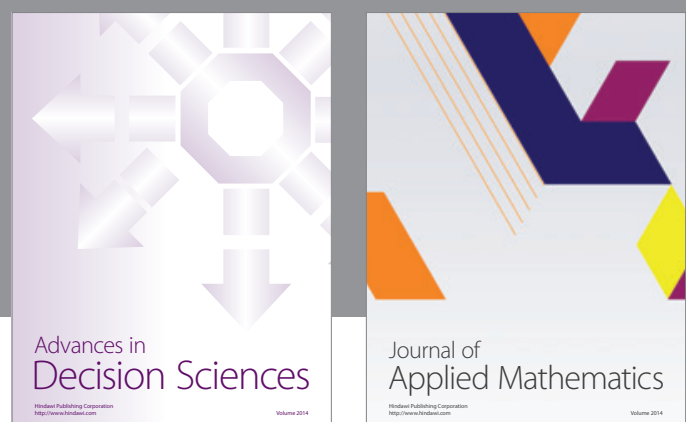

Journal of

Applied Mathematics
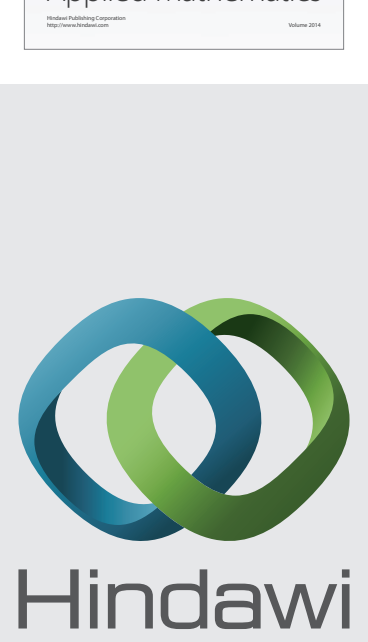

Submit your manuscripts at http://www.hindawi.com
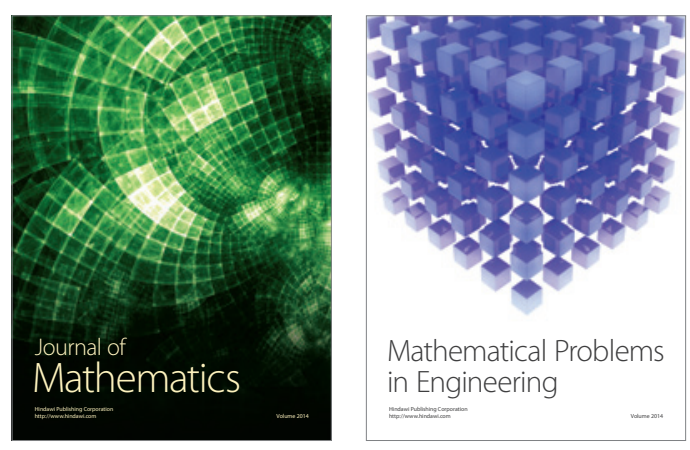

Mathematical Problems in Engineering
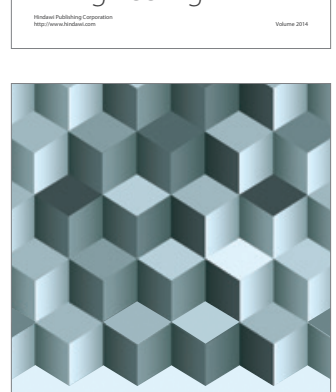

Journal of

Function Spaces
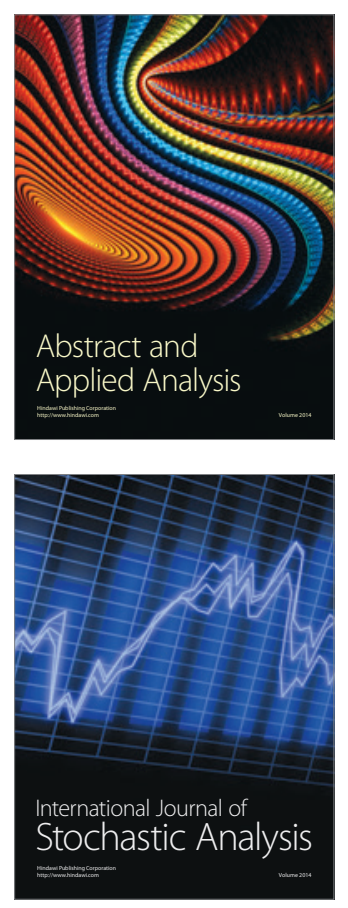

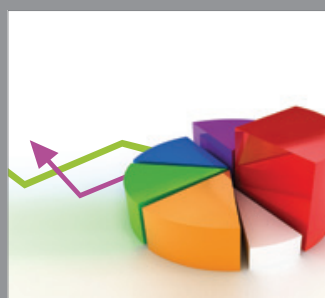

ournal of

Probability and Statistics

Promensencen
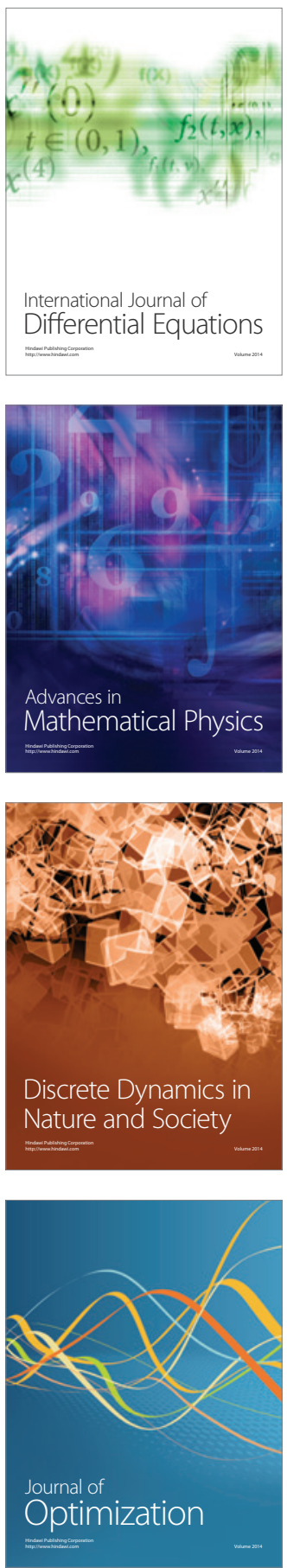